\title{
Influence of completely supra-annular placement of bioprostheses on exercise hemodynamics in patients with a small aortic annulus
}

Ina M. Wagner, MD, Walter B. Eichinger, MD, Sabine Bleiziffer, MD, Florian Botzenhardt, MD, Isabel Gebauer, MD, Ralf Guenzinger, MD, Robert Bauernschmitt, MD, and Ruediger Lange, MD

From the German Heart Center Munich, Clinic of Cardiovascular Surgery, Munich, Germany.

Walter Eichinger reports lecture fees from Edwards and St. Jude. Ruediger Lange reports lecture fees from Edwards and Sorin. Received for publication Aug 3, 2006; revisions received Oct 10, 2006; accepted for publication Oct 23, 2006.

Address for reprints: Ina M. Wagner, MD, Deutsches Herzzentrum München, Klinik für Herz-und Gefäßchirurgie, Lazarettstr. 36, D-80636 München (E-mail: wagner@ dhm.mhn.de).

J Thorac Cardiovasc Surg 2007;133:1234-41 $0022-5223 / \$ 32.00$

Copyright () 2007 by The American Association for Thoracic Surgery

doi:10.1016/j.jtcvs.2006.10.074
Objective: Aortic valve replacement in patients with a small aortic annulus is often associated with increased pressure gradients. For this reason, prostheses for completely supra-annular placement have been developed. To evaluate the potential benefit of this design, the present study compared the effectiveness of 1 intra-supraannular bioprosthesis and 3 completely supra-annular bioprostheses in patients with an aortic annulus diameter of $23 \mathrm{~mm}$ or less.

Methods: Between August 2000 and December 2004, each of 192 patients requiring aortic valve replacement with an intraoperatively measured aortic annulus diameter of $23 \mathrm{~mm}$ or less received one of the following bioprostheses: the stented bovine Sorin Soprano bioprosthesis $(\mathrm{n}=28)$ (Sorin Group, Saluggia, Italy), the Carpentier-Edwards Perimount bioprosthesis $(\mathrm{n}=50)$ (Edwards Lifesciences, Irvine, Calif), the Carpentier-Edwards Perimount Magna bioprosthesis $(\mathrm{n}=70)$ (Edwards Lifesciences), or the stented porcine Medtronic Mosaic $(n=44)$ (Medtronic Inc, Minneapolis, Minn) bioprosthesis. After 6 months, hemodynamic data at rest and during exercise were obtained by echocardiography in 142 patients.

Results: The pericardial valves showed lower mean systolic pressure gradients, larger effective orifice areas and indices, and superior effective orifice fractions than did the porcine valve $(P<.05)$ (Carpentier-Edwards Perimount: $10.9 \pm 3.6 \mathrm{~mm}$ $\mathrm{Hg}, 1.59 \pm 0.41 \mathrm{~cm}^{2}, 0.9 \pm 0.25 \mathrm{~cm}^{2} / \mathrm{m}^{2}, 41.9 \% \pm 9.6 \%$; Carpentier-Edwards Perimount Magna $10.1 \pm 3.8 \mathrm{~mm} \mathrm{Hg}, 1.64 \pm 0.38 \mathrm{~cm}^{2}, 0.93 \pm 0.22 \mathrm{~cm}^{2} / \mathrm{m}^{2}, 45.1 \%$ $\pm 10.2 \%$; Sorin Soprano $13.5 \pm 5.0 \mathrm{~mm} \mathrm{Hg}, 1.64 \pm 0.32 \mathrm{~cm}^{2}, 0.92 \pm 0.15 \mathrm{~cm}^{2} / \mathrm{m}^{2}$, $45.8 \% \pm 9.0 \%$; vs Medtronic Mosaic $15.5 \pm 5.2 \mathrm{~mm} \mathrm{Hg}, 1.31 \pm 0.42 \mathrm{~cm}^{2}, 0.75 \pm$ $0.24 \mathrm{~cm}^{2} / \mathrm{m}^{2}, 35.2 \% \pm 10.0 \%$, respectively). The lowest mean systolic pressure gradients were found after the implantation of the Carpentier-Edwards Perimount Magna. Effective orifice areas, indices, and fractions of the pericardial valves did not show significant differences.

Conclusions: In patients with small aortic roots, transvalvular gradients and effective orifice area showed a tendency to superior results in pericardial valves compared with the porcine bioprosthesis. However, the completely supra-annular design does not necessarily lead to superior hemodynamic results compared with the intra-supra-annular position.

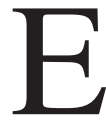
ven with the most sophisticated design of prostheses for aortic valve replacement, a residual pressure gradient may remain, especially in patients with a small aortic annulus or aortic root.

A new concept for optimizing hemodynamic performance is the construction of prostheses that are designed for completely supra-annular implantation. The stent material of completely supra-annular valves is placed on top of the sewing ring. Ventriculoarterial mattress sutures combined with the new design theoretically 


\section{Abbreviations and Acronyms \\ $\mathrm{EOA}=$ effective orifice area \\ EOAI $=$ effective orifice area index \\ $\mathrm{EOF}=$ effective orifice fraction \\ LVOT $=$ left ventricular outflow tract \\ PPM = patient-prosthesis mismatch \\ VTI = velocity time integral}

ensure that stent and sewing ring material do not reach the outflow tract, thus impairing the flow of blood. This design was first implemented in the Medtronic Mosaic (Medtronic Inc, Minneapolis, Minn) bioprosthesis, followed by the Carpentier-Edwards Perimount Magna (Edwards Lifesciences, Irvine, Calif) and Sorin Soprano (Sorin Group, Saluggia, Italy) bioprostheses.

This study evaluates the systolic pressure gradients and effective orifice areas (EOA) of these valves in patients with a small aortic annulus at rest and during exercise, and analyzes the potential hemodynamic benefit resulting from completely supra-annular placement. As a reference valve with an intra-supra-annular design, the Carpentier-Edwards Perimount (Edwards Lifesciences) pericardial prosthesis was included.

For hemodynamic comparisons of different tissue prostheses, the labeled valve sizes may not be suitable, because there is a discrepancy between geometric dimensions and valve size labeling by different companies. ${ }^{1-4}$ The bias that is caused by using the labeled valve size can be avoided by using an independent index for all valve types, which refers to the intraoperatively measured aortic annulus area and the valve's hemodynamic performance. The independent index we use is the effective orifice fraction (EOF), which was previously introduced by our group. ${ }^{3}$

\section{Materials and Methods \\ Bioprostheses}

The Carpentier-Edwards Perimount (Perimount) valve is a stented bovine pericardial bioprosthesis for intra-supra-annular implantation. It received Food and Drug Administration approval in 1991. High rates of freedom from valve-related adverse events have been shown in long-term studies. ${ }^{5}$

The Carpentier-Edwards Perimount Magna (Magna) valve is a stented bovine pericardial bioprosthesis. The valve corresponds to the Perimount in terms of tissue preparation and prosthetic material, but it has been modified to permit completely supra-annular placement. In addition, this valve has a reduced sewing ring. The Magna prosthesis has been in clinical use since September 2002.

The Medtronic Mosaic (Mosaic) valve is a stented porcine bioprosthesis. It has been in clinical use in Europe since 1994 and in the United States since 2000 and has exhibited high rates of freedom from adverse events in midterm follow-up studies. ${ }^{6-8}$

The Sorin Soprano (Soprano) valve is a stented bovine bioprosthesis. In terms of tissue preparation and prosthetic material, it corresponds to the Sorin Pericarbon More, which has been in clinical use for more than 15 years ${ }^{9}$ but differs in stent design. It is designed to permit completely supra-annular implantation to improve hemodynamic performance. It has been in clinical use since August 2003.

\section{Surgical Procedure and Measurement of the Aortic Annulus With a Universal Metric Sizer}

Aortic valve replacement was performed using standard cardiopulmonary bypass with moderate hypothermia at $32^{\circ} \mathrm{C}$ with cold crystalloid cardioplegic cardiac arrest. After removal of the native aortic valve and decalcification of the aortic annulus and root, the internal diameter of the aortic annulus was routinely measured by inserting a probe (Hegar dilator) into the annulus (unit: $1 \mathrm{~mm}$ ). With the assumption that the aortic annulus approaches a circular shape, the annulus orifice area was calculated as follows: (Hegar dilator's diameter $[\mathrm{cm}] \times 0.5)^{2} \times \Pi$. The prosthetic valve size was determined by using the original sizer provided by each manufacturer. All bioprostheses were implanted with pledgeted, interrupted, non-everting mattress sutures.

\section{Completely Supra-annular Placement}

Completely supra-annular placement is achieved by using ventriculoarterial mattress sutures combined with the new valve design, in which the stent is placed on top of the sewing ring and not beside the sewing ring.

\section{Patient Enrollment and Follow-up}

Between August 2000 and December 2004, 192 patients with an aortic stenosis or combined aortic lesion diagnosis requiring valve replacement entered the study. Only patients with an intraoperatively measured aortic annulus diameter of $23 \mathrm{~mm}$ or less were included in this prospective, nonrandomized study, irrespective of the labeled valve size of the implanted prosthesis. Fifty patients received the Perimount valve, 70 patients received the Perimount Magna valve, 44 patients received the Mosaic valve, and 28 patients received the Soprano valve. At our institution, the 4 tested valves were not all on hand at the same time during the whole period of the study. The Mosaic and Perimount valves have been used since August 2000. They were followed by the Magna in January 2001 and the Soprano in September 2003. We included the patients consecutively to avoid bias by patient selection; the consequence is the variance in group size. The valve type was chosen according to the surgeon's preference. All patients gave informed consent to participate in the study. The study was approved by the local ethics committee. Concomitant procedures were allowed, except for valve replacement in another position. Patients requiring valve replacement for acute endocarditis or emergency cases were not included in the study. Body surface area, aortic annulus diameter, and patient age and gender at the time of operation are summarized in Table 1. There is no significant difference between the groups.

Hemodynamic data obtained 6 months postoperatively during rest and mild physical exercise on a bicycle ergometer were available in 142 patients.

The reasons for nonattendance at the follow-up examination are given in Table 2. There is no difference between the groups who finally underwent the stress test (Table 3). 
TABLE 1. Patients' characteristics at the time of operation

\begin{tabular}{lccccc}
\hline & $\begin{array}{c}\text { Perimount (Edwards } \\
\text { Lifesciences, Irvine, Calif) }\end{array}$ & $\begin{array}{c}\text { Magna (Edwards } \\
\text { Lifesciences) }\end{array}$ & $\begin{array}{c}\text { Mosaic (Medtronic Inc, } \\
\text { Minneapolis, Minn) }\end{array}$ & $\begin{array}{c}\text { Soprano (Sorin Group, } \\
\text { Saluggi, Italy) }\end{array}$ & $\boldsymbol{P}$ \\
\hline No. of patients & 50 & 70 & 44 & 28 & \\
Age at implant (y) & $75.2 \pm 6.1$ & $74.2 \pm 6.2$ & $75.9 \pm 5.6$ & $73.9 \pm 6.1$ & .705 \\
Range & $60.1-87.8$ & $55.2-84.9$ & $65.6-89.4$ & $61.1-83.6$ & .297 \\
Gender M/F (\% M) & $13 / 37(26)$ & $24 / 46(34)$ & $13 / 31(30)$ & $13 / 15(46)$ & .298 \\
Annulus diameter (mm) & $21.90 \pm 1.20$ & $21.46 \pm 1.49$ & $21.61 \pm 1.15$ & $21.39 \pm 1.34$ & .703 \\
BSA (m²) & $1.75 \pm 0.20$ & $1.76 \pm 0.19$ & $1.74 \pm 0.16$ & $1.78 \pm 0.16$ &
\end{tabular}

$B S A$, Body surface area.

\section{Echocardiographic Measurements and Calculations}

The echocardiographic examinations were exclusively performed by two experienced echocardiographers. Echocardiographic measurements performed at rest included transvalvular flow velocity, pressure gradient, and velocity time integral (VTI) using continuouswave Doppler. Pulsed-wave Doppler was used for the measurements in the left ventricular outflow tract (LVOT). LVOT diameter was assessed from a parasternal long-axis view using an expanded (zoom) view. The same measurements were performed during exercise, except for LVOT diameter, which was assumed to remain constant. ${ }^{10}$ The mean systolic pressure gradient was calculated as the difference of mean aortic and mean LVOT gradient.

The EOA was calculated by using the continuity equation:

$\operatorname{EOA}\left[\mathrm{cm}^{2}\right]$

$=(\text { LVOT diameter }[\mathrm{cm}] \times 0.5)^{2} \times \Pi \times \mathrm{VTI}_{\mathrm{LVOT}} / \mathrm{VTI}_{\mathrm{valve}}$

The effective orifice area index (EOAI $\left[\mathrm{cm}^{2} / \mathrm{m}^{2}\right]$ ) was calculated by dividing the EOA by body surface area (DuBois formula). ${ }^{11}$

The $\mathrm{EOF}^{3}$ was used to compare the different valve types. This is the relation of Doppler-derived EOA to the anatomic annulus area measured intraoperatively.

$\mathrm{EOF}=$ Doppler-derived EOA $\left[\mathrm{cm}^{2}\right] /$ anatomic annulus area $\left[\mathrm{cm}^{2}\right]$.

\section{Exercise Protocol}

During bicycle exercise (Ergoline, ergometrics er900EL, Bitz, Germany), patients sat reclined in a 50-degree position. After the patients started exercise with a workload of 25 Watts, the workload was increased by 25 Watts every 2 minutes. The patients were encouraged to exercise until exhaustion. In case of unsatisfactory Doppler signals, the whole bicycle unit was tilted to the left side until optimal measurements were obtained. Measurements were performed at the end of each 2-minute workload level. Blood pressure was measured every 2 minutes using a sphygmomanom- eter cuff fixed on the right arm. A 12-lead electrocardiogram was continuously recorded. Exercising was stopped by the investigator in case of hypertension (blood pressure $>210 / 110 \mathrm{~mm} \mathrm{Hg}$ ), electrocardiogram changes as ST segment abnormalities, new arrhythmias (atrial fibrillation, premature ventricular complexes as couplets, bigemini, or trigemini), tachycardia with more than 120 beats/min, angina pectoris, or severe dyspnea.

\section{Patient-Prosthesis Mismatch}

According to Blais and colleagues, ${ }^{12}$ patient-prosthesis mismatch (PPM) was defined as severe if the prosthetic aortic valve EOAI was $0.65 \mathrm{~cm}^{2} / \mathrm{m}^{2}$ or less, as moderate if EOAI was greater than $0.65 \mathrm{~cm}^{2} / \mathrm{m}^{2}$ and $0.85 \mathrm{~cm}^{2} / \mathrm{m}^{2}$ or less, and as clinically not significant if EOAI was greater than $0.85 \mathrm{~cm}^{2} / \mathrm{m}^{2}$. To calculate the rate of PPM, the body surface area and the Doppler-derived EOA were assessed 6 months postoperatively in every individual patient. EOAs for the prosthetic valves that are available in the literature or provided by the manufacturers were not used for reference.

\section{Statistical Analysis}

Statistical analysis was performed with the Statistical Package for the Social Sciences 14.0 for Windows (SPSS Inc, Chicago, Ill). Continuous data are presented as mean \pm standard deviation. To compare categoric data, the chi-square test was used. To compare continuous data in more than 2 groups, the Kruskal-Wallis test was used. To detect differences between groups, analysis of variance with Bonferroni correction for multiple testing was applied.

\section{Results}

\section{Hemodynamic Results}

Mean systolic pressure gradients and stroke volumes are shown in Figure 1. Gradients increased with every exercise level because of larger stroke volumes. The pericardial valves showed lower gradients compared with the porcine

TABLE 2. Reasons for nonattendance at the follow-up examination 6 months postoperatively

\begin{tabular}{lllll}
\hline & Perimount & Magna & Mosaic & Soprano \\
\hline No hemodynamic data could be obtained after 6 mo & $\mathrm{n}=20(40.0 \%)$ & $\mathrm{n}=15(21.4 \%)$ & $\mathrm{n}=12(27.3 \%)$ & $\mathrm{n}=3(10.7 \%)$ \\
Patient's death & $\mathrm{n}=1$ & $\mathrm{n}=3$ & $\mathrm{n}=1$ & $\mathrm{n}=0$ \\
Refusal to attend follow-up & $\mathrm{n}=15$ & $\mathrm{n}=10$ & $\mathrm{n}=6$ & $\mathrm{n}=3$ \\
Bad sonic conditions & $\mathrm{n}=4$ & $\mathrm{n}=2$ & $\mathrm{n}=5$ & $\mathrm{n}=0$ \\
\hline
\end{tabular}


TABLE 3. Characteristics of patients who underwent stress echocardiography

\begin{tabular}{|c|c|c|c|c|c|}
\hline & Perimount & Magna & Mosaic & Soprano & $P$ \\
\hline Age at implant (y) & $76.15 \pm 6.35$ & $74.98 \pm 6.08$ & $75.30 \pm 5.57$ & $73.39 \pm 5.85$ & .427 \\
\hline Gender M/F (\% M) & $9 / 20(31)$ & $12 / 14(46)$ & $11 / 21(34)$ & $7 / 9(44)$ & .689 \\
\hline Annulus diameter (mm) & $21.93 \pm 1.16$ & $21.81 \pm 1.33$ & $21.69 \pm 1.03$ & $21.25 \pm 1.24$ & .266 \\
\hline $\mathrm{BSA}\left(\mathrm{m}^{2}\right)$ & $1.78 \pm 0.20$ & $1.81 \pm 0.22$ & $1.77 \pm 0.22$ & $1.74 \pm 0.15$ & .702 \\
\hline
\end{tabular}

$B S A$, Body surface area.

valve $(P<.05)$. In addition, the Magna showed lower gradients than did the Soprano and Perimount $(P<.05)$.

The mean EOFs of the 4 valves assessed in this study are shown in Figure 2.

The mean EOAs and mean EOAIs are summarized in Table 4. The Mosaic had a significantly lower EOA and EOAI compared with those of the 3 other valves $(P<.05)$, whereas no significant difference could be shown between the other valves.

\section{Sizing Results}

The individual intraoperatively measured annulus size (Hegar) and the labeled size for each valve are shown in Table 5.

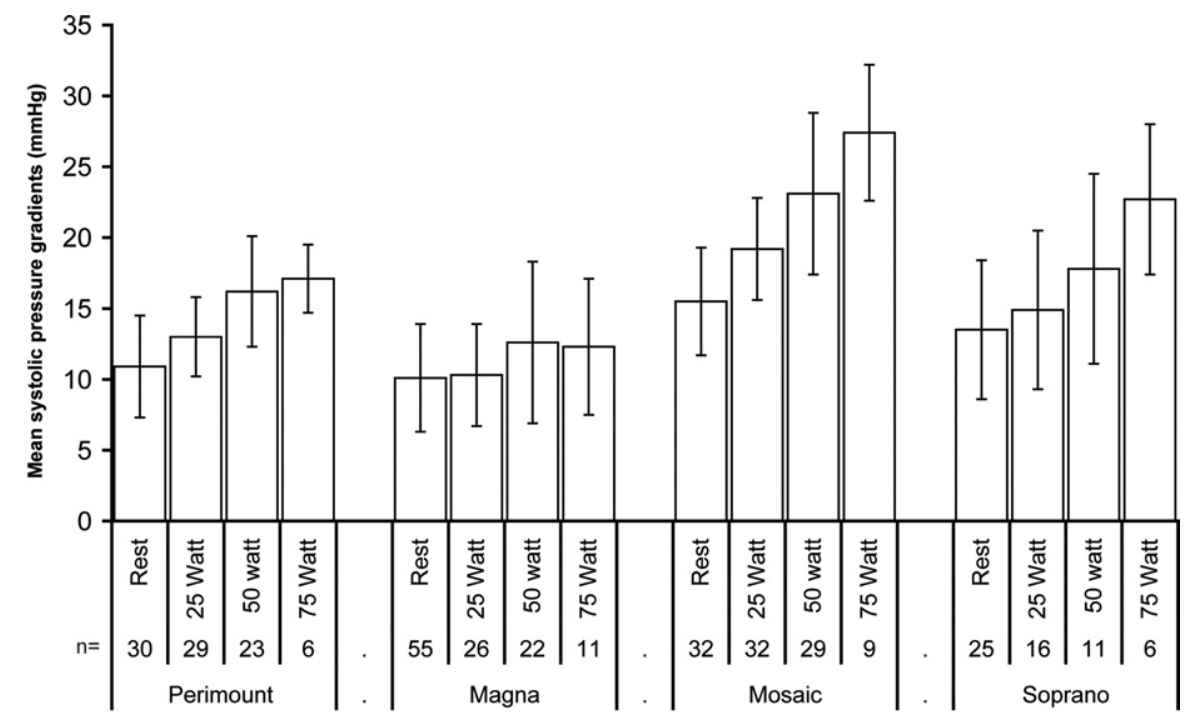

Figure 1. Mean systolic pressure gradients and stroke volumes at rest and during exercise.

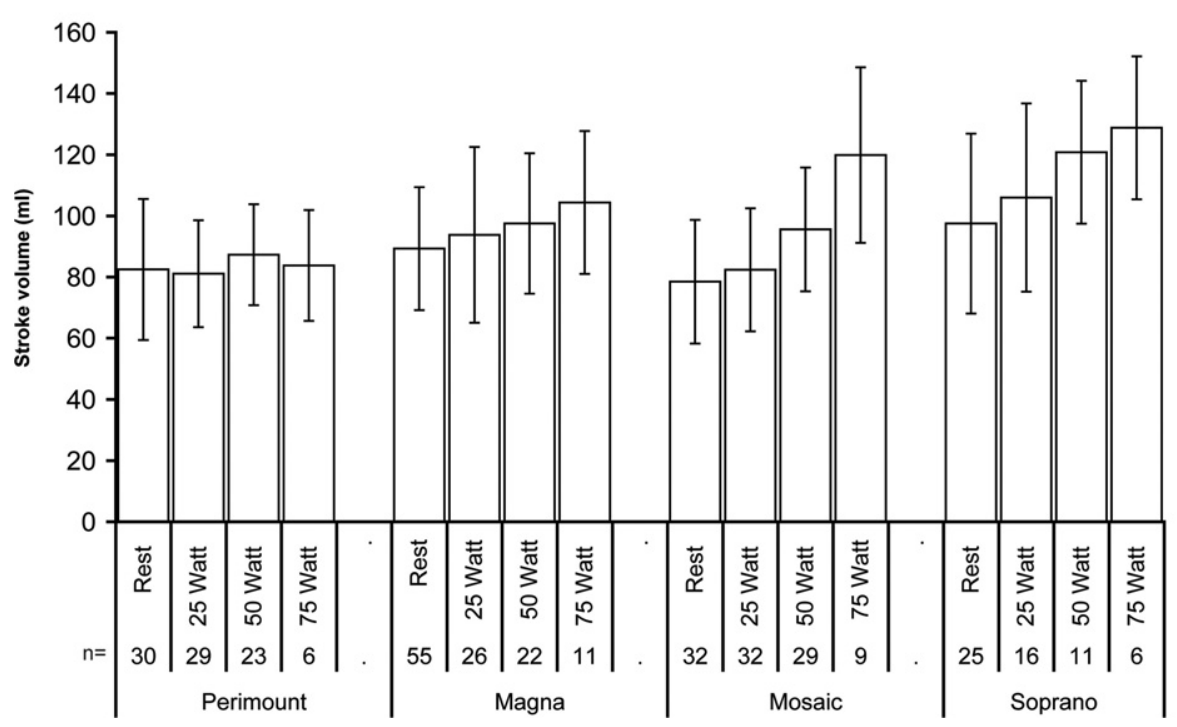




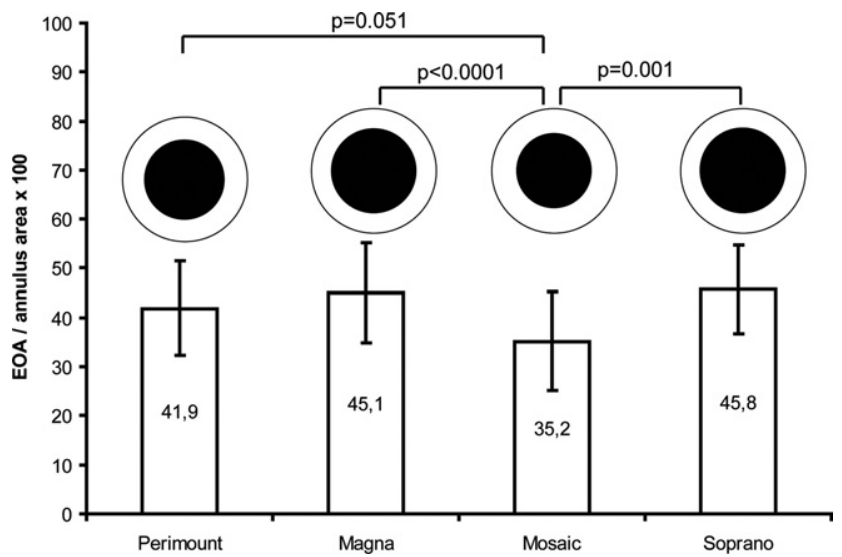

Figure 2. EOF, the independent index for valve performance, of the stented bioprostheses as percentages in the diagram. The mean EOAs (inner black circle) in relation to the annulus areas (outer white circle). EOA, effective orifice area.

\section{Patient-Prosthesis Mismatch}

Figure 3 shows the incidence of PPM in the 4 groups. Six months postoperatively, the incidence of severe PPM in the Magna (3/55) and Soprano (1/25) groups was low. In contrast, 53\% of the patients (16/30) in the Perimount group and $69 \%$ of the patients (22/32) in the Mosaic group showed moderate or severe PPM.

\section{Exercise Testing}

Exercise testing was performed in 106 of 142 patients. The reasons why patients could not perform exercise testing were orthopedic ailments (back, hip, knee pain) $(n=27)$, peripheral arterial occlusive disease $(n=2)$, disability caused by hemiplegia $(n=2)$, New York Heart Association functional class III $(\mathrm{n}=3)$, hypertension at rest $(\mathrm{n}=2)$, and refusal $(\mathrm{n}=3)$. Because of bad sonic conditions in 3 patients, data during exercise could not be obtained. Termination by the investigator because of the reasons mentioned in the "Methods" section was necessary in 16 patients. Eleven patients continued up to 100 Watts. Twenty-six patients stopped exercising because of peripheral pain, 4 patients stopped because of peripheral arterial occlusive disease, and the others stopped because of arthrosis-related knee or hip pain. Twelve patients terminated exercising because of leg muscle weakness, and 16 patients terminated exercising because of peripheral exhaustion. Twenty-five patients stopped exercising because of cardiopulmonary exhaustion.

\section{Discussion}

\section{Hemodynamic Performance}

The systolic hemodynamic performance of the 3 bovine prostheses (Soprano, Magna, and Perimount) and the porcine (Mosaic) bioprosthesis corresponded to the outcome of other reports on these prosthetic heart valves. ${ }^{6,8,13-17}$

Up to now there has been no direct in vivo comparison between the 3 bovine valves and the porcine valve at rest and during exercise. Assessment of hemodynamic variables during exercise may be more appropriate to study the performance of the valves,${ }^{18}$ because it reflects more closely the situation in daily life.

Compared with the other prostheses, the Magna showed the lowest gradients at rest and during exercise. All 3 pericardial valves exhibited lower gradients compared with the porcine valve. We are aware that the measurement of gradients does not entirely represent the complex function of a valve. For this reason we think that in vitro studies (eg, Gerosa and associates ${ }^{19}$ ) performed with the same valves are absolutely necessary and complete our in vivo findings to describe variables such as stroke work loss, total regurgitant, and leakage volumes for the individual prostheses.

In most patients, stroke volume increases during mild exercise. Because of systolic or diastolic dysfunction, mitral valve disease, or elevated afterload and peripheral vascular resistance, the increase of stroke volume may be impaired, especially in elder patients. This could be an explanation for the missing increase of stroke volume at the 75-Watt level in the patients with the Perimount valve.

\section{Comparison of Different Valve Types}

The hemodynamic comparison of different prostheses is complicated by the fact that the labeled valve size does not reflect the same dimensions for each prosthesis and thus is not suitable as a basis for valve comparisons. Prostheses carrying the same labeled valve size may have a different effect on blood flow according to their individual geometric dimensions. ${ }^{1,20}$ To correct for these differences in valve design, comparisons should be based on the EOF as an independent index of valve performance. ${ }^{3}$

TABLE 4. Doppler-derived effective orifice area and effective orifice area index 6 months postoperatively

\begin{tabular}{|c|c|c|c|c|}
\hline & Perimount & Magna & Mosaic & Soprano \\
\hline $\mathrm{EOA} \mathrm{cm}^{2}$ & $1.59 \pm 0.41$ & $1.64 \pm 0.38$ & $1.31 \pm 0.42$ & $1.64 \pm 0.32$ \\
\hline $\mathrm{EOAI} \mathrm{cm}^{2} / \mathrm{m}^{2}$ & $0.90 \pm 0.25$ & $0.93 \pm 0.22$ & $0.75 \pm 0.24$ & $0.92 \pm 0.15$ \\
\hline
\end{tabular}

No significant differences among Perimount, Magna, and Soprano valves for EOA and EOAl; significant differences among Perimount, Magna, and Soprano valves versus Mosaic valve $(P<.05)$ for EOA and EOAI. EOA, Effective orifice area; EOAl, effective orifice area index. 
TABLE 5. Individual intraoperatively measured annulus diameters and the corresponding labeled valve sizes

\begin{tabular}{|c|c|c|c|c|c|c|c|c|c|}
\hline & \multirow[b]{2}{*}{ Valve label } & \multirow[b]{2}{*}{$\mathbf{n}$} & \multicolumn{7}{|c|}{ Annulus diameter (Hegar measurement) } \\
\hline & & & $17 \mathrm{~mm}$ & $18 \mathrm{~mm}$ & $19 \mathrm{~mm}$ & $20 \mathrm{~mm}$ & $21 \mathrm{~mm}$ & $22 \mathrm{~mm}$ & $23 \mathrm{~mm}$ \\
\hline \multirow[t]{4}{*}{ Perimount } & 19 & 4 & & & 2 & 2 & & & \\
\hline & 21 & 24 & & & & 3 & 11 & 7 & 3 \\
\hline & 23 & 21 & & & & & & 3 & 18 \\
\hline & 25 & 1 & & & & & & & 1 \\
\hline \multirow[t]{4}{*}{ Magna } & 19 & 8 & 1 & 1 & 4 & 2 & & & \\
\hline & 21 & 32 & & & & 11 & 17 & 4 & \\
\hline & 23 & 29 & & & & & 1 & 2 & 26 \\
\hline & 25 & 1 & & & & & & & 1 \\
\hline \multirow[t]{4}{*}{ Mosaic } & 19 & 3 & & 1 & & 2 & & & \\
\hline & 21 & 19 & & & & 5 & 7 & 7 & \\
\hline & 23 & 22 & & & & & 2 & 10 & 10 \\
\hline & 25 & & & & & & & & \\
\hline \multirow[t]{4}{*}{ Soprano } & 18 & 3 & & 1 & 1 & 1 & & & \\
\hline & 20 & 16 & & & 1 & 2 & 7 & 3 & 3 \\
\hline & 22 & 8 & & & & & & 5 & 3 \\
\hline & 24 & 1 & & & & & & 1 & \\
\hline
\end{tabular}

In our series the pericardial valves showed a larger EOF compared with the porcine valve. Although the completely supra-annular design should result in an optimized functional use of the anatomically given annulus area, the intra-supra-annular Perimount shows lower mean pressure gradients than the Mosaic and Soprano and no significant difference in EOF compared with the completely supraannular Soprano and Magna. In an in vitro study, Gerosa and colleagues ${ }^{19}$ compared 3 pericardial bioprostheses (Perimount Magna, Sorin Soprano, and Sorin Mitroflow) and 2 porcine bioprostheses (St Jude Medical Epic Supra [St Jude Medical Inc, St Paul, Minn] and Medtronic Mosaic) by using a pulse duplicator. Accordant to our findings, the pericardial valves showed lower pressure gradients and larger EOAs than those of the porcine valves. The authors take into account that a meaningful hemodynamic comparison of different valve types should not be based on industry-labeled valve sizes by comparing prostheses that can fit in a $21-\mathrm{mm}$ pulse duplicator ring regardless of industrylabeled valve size. In vivo, the decision that leads to the selection of a certain valve size is additionally influenced by the complex anatomic structure of the aortic root in combination with prosthesis dimensions, including stent height, sewing ring diameter, completely supra-annular position, and anatomic relation to the coronary ostia (Figure 4).

\section{Patient-Prosthesis Mismatch}

Although the clinical impact of PPM after aortic valve replacement is still considered to be controversial, there is evidence that PPM might lead to higher rates of cardiac morbidity and mortality. ${ }^{12,21-23}$ For the 4 valve types studied, we demonstrated differences in the rates of PPM as defined by Blais and colleagues, ${ }^{12}$ calculated with the Dop- pler-derived EOAI 6 months postoperatively. This graduation corresponds to the assumption that moderate aortic stenosis of a native valve is present if EOAI is less than 0.90 $\mathrm{cm}^{2} / \mathrm{m}^{2}$. ${ }^{24}$ There is general agreement that the only parameter to define PPM is the relation of EOA to the patient's body surface area, but there is still no general agreement on how to calculate the prosthesis EOA.

In our opinion the EOA of an implanted valve is a functional parameter that probably depends on leaflet compliance, orientation, and position (angle) in relation to the LVOT and the ascending aorta. Thus, the EOA has to be assessed for every individual patient and his/her valve substitute. The easiest way to determine the EOA of an implanted valve is Doppler echocardiography with the use of

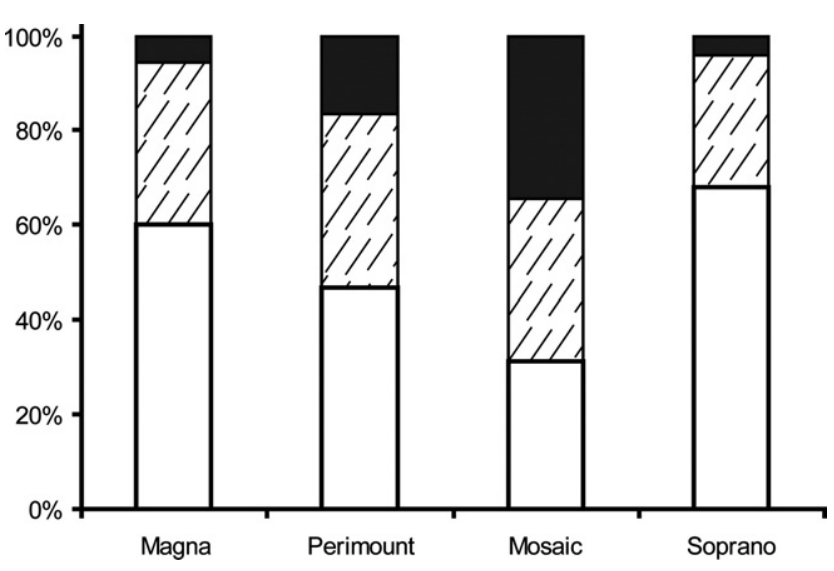

Figure 3. Incidence of PPM calculated with the Doppler-derived EOA 6 months postoperatively. $\square$ severe PPM moderate PPM $\square$ no PPM. PPM, Patient-prosthesis mismatch; EOA, effective orifice area. 


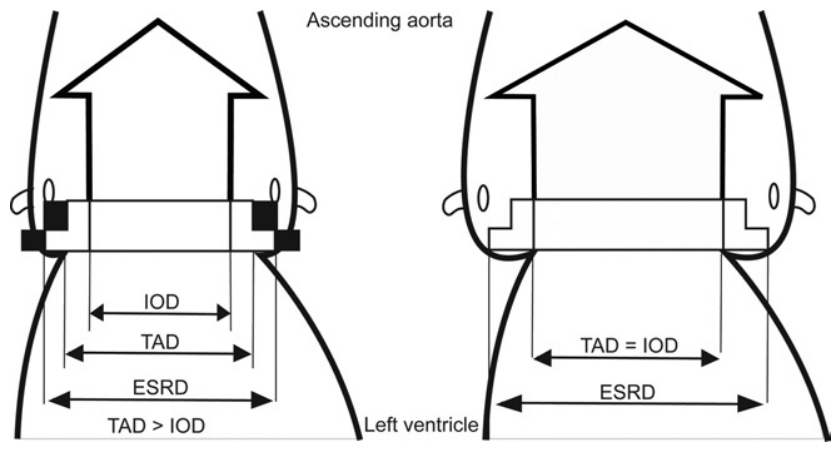

Figure 4. Two aortic root anatomies with an identical TAD. A more bulbar-shaped root (right). This is the ideal situation for completely supra-annular placement in which the tissue annulus diameter corresponds to the internal orifice diameter of the prosthesis (TAD = IOD). Small aortic root (left). Despite the same tissue annular diameter, the valve of the same size (black) does not fit. Thus, the surgeon has to choose a smaller valve (white), with the consequence that despite implantation of a completely supra-annular prosthesis, the stent and sewing ring material impair the bloodstream because the shape of the aortic root does not allow the implantation of a valve large enough to ensure that the IOD corresponds to the TAD. This illustrates the hypothesis that a completely supra-annular prosthesis placement does not ensure a hemodynamic benefit in every aortic root. Even after implantation of a completely supra-annular prosthesis, flow obstruction caused by the stent and sewing ring material may occur if there is a narrow aortic root. TAD, Tissue annulus diameter; IOD, internal orifice diameter; ESRD, external sewing ring diameter.

hydrodynamic principles (continuity equation). However, because the Doppler-derived EOA depends on various hemodynamic conditions, it should not be determined just in the early postoperative period when patients may be still anemic; left ventricular function may not yet have been restored, and volume status may be disordered. In the present study, EOA and the occurrence of PPM were assessed 6 months postoperatively by means of Doppler EOA measurement. According to a literature survey by Pibarot and colleagues, ${ }^{25}$ PPM is present in up to $20 \%$ to $70 \%$ of patients with a stented aortic bioprosthesis. In our series PPM ranged from 32\% (Soprano) to 69\% (Mosaic).

\section{Completely Supra-annular Design}

The new generation of stented bioprostheses for completely supra-annular placement is represented by the Magna, Mosaic, and Soprano valves in our series. This technical development aims at optimizing the systolic hemodynamic performance of any stented prosthesis. The EOF, which represents the use of the anatomically given annulus area, ranged from $35.2 \%$ (Mosaic) to $45.8 \%$ (Soprano) in our series. This reveals that despite the completely supra-annu- lar design, the whole annulus area is not available for blood flow, presumably because the stent and sewing ring material are still positioned within the blood flow. Thus, the completely supra-annular design does not necessarily lead to superior hemodynamic results compared with the intrasupra-annular valve. Presumably, not every aortic root is anatomically suitable to exhibit the advantages of the completely supra-annular design. In a very straight aortic root, with little extension of the sinuses, even the completely supra-annular valve protrudes somewhat into the outflow tract. Figure 4 illustrates our hypothesis that even if a completely supra-annular prosthesis is used, flow obstruction caused by the stent and sewing ring material cannot always be avoided.

\section{Limitations}

Because our study was a nonrandomized study, bias resulting from patient and valve selection by the implanting surgeon cannot be excluded.

The hemodynamic performance of aortic valve substitutes depends on a number of cofactors, such as left ventricular systolic and diastolic function, the patient's volume status, function of the mitral valve, blood pressure, heart rate, and so forth, which were not analyzed in this study.

For various reasons, not all patients were able to reach higher exercise levels. Therefore, the hemodynamic data that are available for comparison at 75 Watts are scarce (Figure 1).

The result of echocardiography may be influenced by the sonic conditions that differ from patient to patient and may therefore be biased.

\section{Conclusions}

The 4 bioprostheses compared in this study showed satisfactory hemodynamic results at rest and during exercise in patients with a small aortic annulus. Completely supraannular valves were designed to minimize the flow obstacle that is caused by the sewing ring and stent material reaching into the annulus diameter. We demonstrated that this concept does not necessarily lead to superior hemodynamic results, which we believe may depend on the anatomy of the aortic root. The pericardial, completely supra-annular Magna bioprosthesis with a reduced sewing ring indicated the lowest pressure gradients at rest and during exercise and seems to deal best with the challenge of achieving good hemodynamics in patients with a small aortic annulus, which is often associated with a narrow ascending aorta.

\section{References}

1. Christakis GT, Buth KJ, Goldman BS, et al. Inaccurate and misleading valve sizing: a proposed standard for valve size nomenclature. Ann Thorac Surg. 1998;66:1198-203. 
2. Botzenhardt F, Eichinger WB, Bleiziffer S, et al. Hemodynamic comparison of bioprostheses for complete supra-annular position in patients with small aortic annulus. J Am Coll Cardiol. 2005;45:2054-60.

3. Eichinger WB, Botzenhardt F, Guenzinger R, et al. The effective orifice area/patient aortic annulus area ratio: a better way to compare different bioprostheses? A prospective randomized comparison of the Mosaic and Perimount bioprostheses in the aortic position. J Heart Valve Dis. 2004;13:382-8; discussion 388-9.

4. Sievers HH. Prosthetic aortic valve replacement. J Thorac Cardiovasc Surg. 2005;129:961-5.

5. Vitale N, Clark SC, Ramsden A, Hasan A, Hilton CJ, Holden MP. Clinical and hemodynamic evaluation of small Perimount aortic valves in patients aged 75 years or older. Ann Thorac Surg. 2003;75:35-9; discussion 40 .

6. Eichinger W, Gunzinger R, Botzenhardt F, Simmerl D, Gansera B, Kemkes BM. The Mosaic bioprosthesis in the aortic position at five years. J Heart Valve Dis. 2000;9:653-60.

7. Eichinger WB, Botzenhardt F, Gunzinger R, et al. Left ventricular mass regression after aortic valve replacement with the mosaic bioprosthesis. J Heart Valve Dis. 2002;11:529-36; discussion 536.

8. Botzenhardt F, Gansera B, Kemkes BM. Mid-term hemodynamic and clinical results of the stented porcine Medtronic mosaic valve in aortic position. Thorac Cardiovasc Surg. 2004;52:34-41.

9. Seguin JR, Grandmougin D, Folliguet T, Warembourg H, Laborde F, Chaptal PA. Long-term results with the Sorin Pericarbon valve in the aortic position: a multicenter study. J Heart Valve Dis. 1998;7:278-82.

10. Rassi A Jr, Crawford MH, Richards KL, Miller JF. Differing mechanisms of exercise flow augmentation at the mitral and aortic valves. Circulation. 1988;77:543-51.

11. DuBois D, DuBois EF. A formula to estimate the approximate surface area if height and weight be known. Arch Intern Med. 1916;17:863-71.

12. Blais C, Dumesnil JG, Baillot R, Simard S, Doyle D, Pibarot P. Impact of valve prosthesis-patient mismatch on short-term mortality after aortic valve replacement. Circulation. 2003;108:983-8.

13. Frater RW, Salomon NW, Rainer WG, Cosgrove DM 3rd, Wickham E. The Carpentier-Edwards pericardial aortic valve: intermediate results. Ann Thorac Surg. 1992;53:764-71.
14. Cosgrove DM, Lytle BW, Williams GW. Hemodynamic performance of the Carpentier-Edwards pericardial valve in the aortic position in vivo. Circulation. 1985;72:II146-52.

15. Takakura H, Sasaki T, Hashimoto K, et al. Hemodynamic evaluation of 19-mm Carpentier-Edwards pericardial bioprosthesis in aortic position. Ann Thorac Surg. 2001;71:609-13.

16. Botzenhardt F, Gansera B, Gunzinger R, Kemkes BM. [Clinical and hemodynamic results of the mosaic bioprosthesis in aortic position]. $Z$ Kardiol. 2003;92:407-14.

17. Eichinger WB, Schutz A, Simmerl D, et al. The mosaic bioprosthesis in the aortic position: hemodynamic performance after 2 years. Ann Thorac Surg. 1998;66:S126-9.

18. Rahimtoola SH. The problem of valve prosthesis-patient mismatch. Circulation. 1978;58:20-4.

19. Gerosa G, Tarzia V, Rizzoli G, Bottio T. Small aortic annulus: the hydrodynamic performances of 5 commercially available tissue valves. J Thorac Cardiovasc Surg. 2006;131:1058-64.

20. Seitelberger R, Bialy J, Gottardi R, et al. Relation between size of prosthesis and valve gradient: comparison of two aortic bioprosthesis. Eur J Cardiothorac Surg. 2004;25:358-63.

21. Blackstone EH, Cosgrove DM, Jamieson WR, et al. Prosthesis size and long-term survival after aortic valve replacement. J Thorac Cardiovasc Surg. 2003;126:783-96.

22. Mohty-Echahidi D, Malouf JF, Girard SE, et al. Impact of prosthesispatient mismatch on long-term survival in patients with small St Jude Medical mechanical prostheses in the aortic position. Circulation. 2006;113:420-6.

23. Walther T, Rastan A, Falk V, et al. Patient prosthesis mismatch affects short- and long-term outcomes after aortic valve replacement. Eur J Cardiothorac Surg. 2006;30:15-9.

24. Rahimtoola SH. Perspective on valvular heart disease: an update. J Am Coll Cardiol. 1989;14:1-23.

25. Pibarot P, Dumesnil JG, Lemieux M, Cartier P, Metras J, Durand LG. Impact of prosthesis-patient mismatch on hemodynamic and symptomatic status, morbidity and mortality after aortic valve replacement with a bioprosthetic heart valve. J Heart Valve Dis. 1998;7:211-8. 